\title{
FAME
}

\section{Fractional Flow Reserve versus Angiography for Guiding PCl in Patients with Multivessel Coronary Artery Disease}

\section{FFR im Vergleich zur angiographisch gesteuerten $\mathrm{PCl}$ bei Patienten mit koronarer Mehrgefäßerkrankung}

Hintergrund und Problemstellung: Das routinemäßige Stenting von Koronarstenosen ohne Ischämienachweis bringt keinen Nutzen, während die Stentversorgung von Stenosen mit Ischämienachweis sowohl die Symptomatik als auch die Prognose verbessern kann. Bei Patienten mit Mehrgefäßerkrankung sind nichtinvasive Tests zur regionalen Zuordnung des Ischämienachweises gelegentlich ungenau und unzuverlässig. Angiographisch kann die funktionelle Bedeutung von Stenosen leicht

\section{Methodik}

Insgesamt wurden 1905 Patienten mit koronarer Mehrgefäßerkrankung gescreent. Nach Ausschluss von 900 Patienten wurden 1005 Patienten randomisiert. In der FFR-Gruppe wurde die FFR mit dem Certus-Druckdraht der Firma RADI Medical Systems nach Gabe von Adenosin in einer Dosis von $140 \mu \mathrm{g} / \mathrm{kg} / \mathrm{min}$ in eine Femoralvene gemessen.

\begin{tabular}{|c|c|}
\hline Studiendesign & Prospektiv, randomisiert, multizentrisch \\
\hline Primärer Endpunkt & $\begin{array}{l}\text { MACE nach } 1 \text { Jahr (Tod, Herzinfarkt oder } \\
\text { erneute Revaskularisation) }\end{array}$ \\
\hline Sekundäre Endpunkte & $\begin{array}{l}\text { Einzelkomponenten von MACE, funktionelle } \\
\text { AP-Klasse, Verbrauch von Antianginosa, } \\
\text { Prozedurdauer, Kontrastmittelverbrauch, } \\
\text { Lebensqualität }\end{array}$ \\
\hline Patientenauswahl & $\begin{array}{l}\text { Einschlusskriterien: } \\
\text { - Alle Patienten mit Mehrgefäßerkrankung } \\
\text { - Mindestens zwei stentbare Stenosen } \geq 50 \% \text { in } \\
\text { zwei oder drei epikardialen Koronargefäßen } \\
\text { Ausschlusskriterien: } \\
\text { - Hauptstammstenose oder vorherige Bypass- } \\
\text { operation } \\
\text { - STEMI mit CK > } 1000 \mathrm{U} / \mathrm{l} \text { in den letzten } \\
5 \text { Tagen } \\
\text { - Extrem torquierte oder kalzifizierte Koronarien }\end{array}$ \\
\hline $\begin{array}{l}\text { Anzahl der teil- } \\
\text { nehmenden Zentren }\end{array}$ & 20 (Europa und USA) \\
\hline
\end{tabular}

nehmenden Zentren

Evidence-based-Medicine-(EBM-)Score

Klinischer primärer Endpunkt

$\mathrm{Ja}=3$

Nein $=0$

$\mathrm{Ja}=1$

Nein $=0$

Doppelblind (einschließlich Ärzten)

Beobachtungsintervall für den primären Endpunkt

$\geq 6$ Monate

Multicenter (mindestens 3 Zentren)

$\mathrm{Ja}=1$

Nein $=0$

$\mathrm{Ja}=1$

Nein $=0$

Externes und vom Steering Committee unabhängiges

Clinical Event Committee/DSMB (Datensicherheit-

Monitoring-Board)

Primärer Endpunkt erreicht $\quad \mathrm{Ja}=1$

Power von $\geq 80 \%$ für den primären Endpunkt erreicht

Anteil des Follow-up der Patienten für

angiographischen primären Endpunkt $\geq 80 \%$ und

$\geq 95 \%$ für klinischen primären Endpunkt

Gesamt-EBM-Score

$\mathrm{Ja}=1$

Nein $=0$

Nein $=0$

$\mathrm{Ja}=1$

Nein $=0$

$\mathrm{Ja}=1$

Nein $=0$

„Silber-Score“ für randomisierte, kontrollierte Studien (RaCT) oder kontrollierte Registerstudien (ReCT mit präspezifizierter Kontrollgruppe und Power-Kalkulation); es sind maximal 10 Scorepunkte möglich (J Interv Cardiol 2006;19:485-92) unterschätzt wie auch überschätzt werden. Im Gegensatz hierzu ist die Bestimmung der fraktionellen Flussreserve (FFR) der selektivste und genaueste Parameter für eine induzierbare Ischämie. Ziel der FAME-Studie war es, festzustellen, ob die FFRgesteuerte PCI bei Patienten mit Mehrgefäßerkrankung einer ausschließlich angiographiegesteuerten PCI überlegen ist. Studienleiter waren N. Pijls, Eindhoven, Niederlande, und W. Fearon, Stanford, CA, USA.

\section{Ergebnisse $\left({ }^{*} p<0,05\right)$}

Insgesamt wurden in der FFR-Gruppe 874 von 1329 Stenosen (63\%) mit einer $F F R \leq 0,80$ mit insgesamt 980 Stents versorgt, während 513 Stenosen $(37 \%)$ nicht gestentet wurden, da die FFR $>0,80$ lag.

In der angiographiegesteuerten Gruppe wurden $92 \%$ aller Stenosen mit insgesamt 1359 Stents versorgt. In beiden Gruppen wurden ausschließlich beschichtete Stents benutzt.

\begin{tabular}{|c|c|c|}
\hline & $\begin{array}{l}\text { Angiographie- } \\
\text { gesteuerte PCI }\end{array}$ & $\begin{array}{l}\text { FFR-gesteuerte } \\
\text { PCI }\end{array}$ \\
\hline Patientenanzahl & $n=496$ & $\mathrm{n}=509$ \\
\hline Patientencharakteristika & \multicolumn{2}{|c|}{$\begin{array}{l}\text { Beide Gruppen waren hinsichtlich der } \\
\text { Ausgangsparameter vergleichbar }\end{array}$} \\
\hline Stenosen pro Patient & $2,7 \pm 0,9$ & $2,8 \pm 1,0$ \\
\hline $\begin{array}{l}\text { Prozedurdaten } \\
\text { Stents pro Patient } \\
\text { Gesamte Stentzahl (DES) } \\
\text { Prozedurdauer (min) } \\
\text { Kontrastmittelverbrauch (ml) }\end{array}$ & $\begin{array}{l}2,7 \pm 1,2 \\
1359 \\
70 \pm 44 \\
302 \pm 127\end{array}$ & $\begin{array}{l}1,9 \pm 1,35^{*} \\
980 \\
71 \pm 43 \\
272 \pm 133^{*}\end{array}$ \\
\hline $\begin{array}{l}\text { Primärer Endpunkt } \\
\text { MACE nach } 1 \text { Jahr (\%) }\end{array}$ & 18,4 & $13,2^{*}$ \\
\hline $\begin{array}{l}\text { Sekundäre Endpunkte } \\
\text { Tod (\%) } \\
\text { Myokardinfarkt (\%) } \\
\text { Erneute Revaskularisation (\%) } \\
\text { Patienten ohne AP (\%) }\end{array}$ & $\begin{array}{r}3,0 \\
8,7 \\
9,5 \\
78\end{array}$ & $\begin{array}{r}1,8 \\
5,7 \\
6,5 \\
81\end{array}$ \\
\hline
\end{tabular}

Schlussfolgerung und Kommentar: Die Ergebnisse der FAMEStudie belegen für den primärem Endpunkt MACE nach $1 \mathrm{Jahr}$ die Überlegenheit der FFR-gesteuerten PCI bei Patienten mit Mehrgefäßerkrankung im Vergleich zur angiographiegesteuerten PCI. Die Ergebnisse von FAME dokumentieren erneut eindrucksvoll die bereits bekannte Tatsache, dass ein unkritisches, routinemäßiges Stenting aller Stenosen $>50 \%$ nach dem angiographiegetriggerten „okulostenotischen Reflex“ für die Patienten von Nachteil ist. Die günstigeren Ergebnisse bei FFR-gesteuerter PCI belegen andererseits aber nicht die Notwendigkeit, die FFR bei Mehrgefäßerkrankung routinemäßig zu messen, da in FAME keine Gruppe mit konventioneller Ischämietestung untersucht wurde. Zukünftige Studien müssen klären, ob und bei welchen Patienten die invasive FFR-Messung zusätzlich zur konventionellen nichtinvasiven Ischämietestung tatsächlich einen Nutzen bringt.

W. Grimm, Marburg

Herz 2008;33:609

DOI $10.1007 / \mathrm{s} 00059-008-3186-1$ 